\title{
Optimized Effective Potential for Quantum Electrodynamical Time-Dependent Density Functional Theory
}

\author{
Camilla Pellegrini, ${ }^{1, *}$ Johannes Flick, ${ }^{2, \dagger}$ Ilya V. Tokatly, ${ }^{1,3,}$ Heiko Appel, ${ }^{2,4, \S}$ and Angel Rubio ${ }^{1,2,4, \|}$ \\ ${ }^{1}$ Departamento de Fisica de Materiales, Nano-bio Spectroscopy Group and ETSF Scientific Development Centre, \\ Universidad del Pais Vasco UPV/EHU, E-20018 San Sebastian, Spain \\ ${ }^{2}$ Fritz-Haber-Institut der Max-Planck-Gesellschaft, Faradayweg 4-6, D-14195 Berlin, Germany \\ ${ }^{3}$ IKERBASQUE, Basque Foundation for Science, 48011 Bilbao, Spain \\ ${ }^{4}$ Max Planck Institute for the Structure and Dynamics of Matter, Luruper Chaussee 149, 22761 Hamburg, Germany
}

(Received 12 December 2014; revised manuscript received 25 May 2015; published 24 August 2015)

We propose an orbital exchange-correlation functional for applying time-dependent density functional theory to many-electron systems coupled to cavity photons. The time nonlocal equation for the electronphoton optimized effective potential (OEP) is derived. In the static limit our OEP energy functional reduces to the Lamb shift of the ground state energy. We test the new approximation in the Rabi model. It is shown that the OEP (i) reproduces quantitatively the exact ground-state energy from the weak to the deep strong coupling regime and (ii) accurately captures the dynamics entering the ultrastrong coupling regime. The present formalism opens the path to a first-principles description of correlated electron-photon systems, bridging the gap between electronic structure methods and quantum optics for real material applications.

The last two decades have witnessed increasing experimental interest in the study and control of many-electron systems strongly interacting with quantum electromagnetic fields. This includes notable experiments in the areas of cavity [1] and circuit [2] quantum electrodynamics (QED), quantum computing via photon-mediated atom entanglement [3], electromagnetically induced transparency [4], quantum plasmonics [5], quantum simulators [6], and chemistry $[7,8]$. The description of realistic coupled matterphoton systems requires combining electronic structure methods from materials science with quantum optical models. Recently, a time-dependent density-functional theory (TDDFT) for QED has been developed [9-12], allowing for such a first-principles treatment. However, any application of this theory requires approximations to the electron-photon exchange-correlation (xc) functional, which are currently not available.

In this Letter we construct the first approximation to the $\mathrm{xc}$ functional of QED-(TD)DFT. To achieve this goal, we extend the widely used optimized effective potential (OEP) approach in electronic structure methods [13-18] to the photon-mediated electron-electron coupling. The new functional is tested from low to high coupling regime in the Rabi model [19-21], through comparison with the exact and classical solutions. We also address the functional dependence on the initial many-body state, assumed to be either a fully interacting or a factorizable state. In both cases, the electron-photon OEP for the model performs well, providing a promising path to the ab initio description of strongly coupled matter-photon systems.

Consider a system with an arbitrarily large number $N$ of electrons at coordinates $\left\{\mathbf{r}_{i}\right\}_{i=1}^{N}$, e.g., an atom, an ion, or a molecule, interacting with $M$ quantized electromagnetic modes of a microcavity with frequencies $\omega_{\alpha}$. We denote by $\hat{H}_{0}=\hat{T}+\hat{V}_{\text {ee }}+\hat{V}_{\text {ext }}$ the Hamiltonian of the electronic system with kinetic energy $\hat{T}$, Coulomb interaction $\hat{V}_{\text {ee }}$, and (time-dependent) external potential $\hat{V}_{\text {ext }}=\sum_{i=1}^{N} v_{\text {ext }}\left(\mathbf{r}_{i} t\right)$, due to the nuclei and any classical field applied to the system. In the dipole approximation [22] the length-gauge Hamiltonian $[9,23,24]$ of the total electron-photon system can be represented as follows:

$$
\hat{H}=\hat{H}_{0}+\frac{1}{2} \sum_{\alpha}\left[\hat{p}_{\alpha}^{2}+\omega_{\alpha}^{2}\left(\hat{q}_{\alpha}-\frac{\lambda_{\alpha}}{\omega_{\alpha}} \hat{\mathbf{R}}\right)^{2}\right],
$$

where the second term corresponds to the energy $1 / 8 \pi \int\left(\hat{\mathbf{B}}^{2}+\hat{\mathbf{E}}^{2}\right) d \mathbf{r}$ of the transverse radiation field. The magnetic field $\hat{B}_{\alpha}=\sqrt{4 \pi} \hat{p}_{\alpha}$ in the $\alpha$ mode is proportional to the photon canonical momentum $\hat{p}_{\alpha}$, while the electric field $\hat{E}_{\alpha}=\sqrt{4 \pi}\left(\omega_{\alpha} \hat{q}_{\alpha}-\lambda_{\alpha} \hat{\mathbf{R}}\right)$ is related to the canonical coordinate $\hat{q}_{\alpha}$. The latter is defined via the displacement field $\hat{D}_{\alpha}=\sqrt{4 \pi} \omega_{\alpha} \hat{q}_{\alpha}$, which is the proper dynamical variable conjugated to $\hat{B}_{\alpha}$. Finally, $\boldsymbol{\lambda}_{\alpha}$ describes the polarization and the amplitude of the normalized $D_{\alpha}$ mode at the position of the electronic system with dipole moment $\hat{\mathbf{R}}=\sum_{i=1}^{\mathbf{N}} \mathbf{r}_{i}$ [25]. We define $\hat{q}_{\alpha}=-\left(\hat{a}_{\alpha}+\hat{a}_{\alpha}^{\dagger}\right) / \sqrt{2 \omega_{\alpha}}$ in terms of photon annihilation and creation operators. The photon-induced interaction Hamiltonian in Eq. (1) consists of two terms: (i) the "cross" term $\sim \hat{q}_{\alpha} \hat{\mathbf{R}}$

$$
\hat{V}_{\mathrm{el}-\mathrm{ph}}=\sum_{\alpha} \sqrt{\frac{\omega_{\alpha}}{2}}\left(\hat{a}_{\alpha}+\hat{a}_{\alpha}^{\dagger}\right) \int d^{3} \mathbf{r}\left(\boldsymbol{\lambda}_{\alpha} \mathbf{r}\right) \hat{n}(\mathbf{r}),
$$


where $\hat{n}(\mathbf{r})=\sum_{i} \delta\left(\mathbf{r}-\mathbf{r}_{i}\right)$ is the electron density operator, describing the dipole-displacement coupling, and (ii) the "squared" term $\sum_{\alpha}\left(\boldsymbol{\lambda}_{\alpha} \hat{\mathbf{R}}\right)^{2} / 2$, representing the polarization energy of the electrons. The coupling to the quantized radiation field then gives rise to an additional, photonmediated electron-electron interaction

$$
\begin{aligned}
W_{\text {ee }}(1,2) & =\sum_{\alpha}\left(\boldsymbol{\lambda}_{\alpha} \mathbf{r}_{1}\right)\left(\boldsymbol{\lambda}_{\alpha} \mathbf{r}_{2}\right) \mathcal{W}_{\alpha}\left(t_{1}, t_{2}\right), \\
\mathcal{W}_{\alpha}\left(t_{1}, t_{2}\right) & =\omega_{\alpha}^{2} \mathcal{D}_{\alpha}\left(t_{1}, t_{2}\right)+\delta\left(t_{1}-t_{2}\right),
\end{aligned}
$$

where we use the compact notation $1=\left(\mathbf{r}_{1} t_{1}\right)$. Here, the first term is the effective interaction, via the photon displacement $D_{\alpha}$ propagator $\mathrm{i} \mathcal{D}_{\alpha}\left(t_{1}, t_{2}\right) \equiv\left\langle\mathcal{T}\left\{q_{\alpha}\left(t_{1}\right) q_{\alpha}\left(t_{2}\right)\right\}\right\rangle$, derived from Eq. (2). The second term, due to the polarization contribution in the Hamiltonian, removes the instantaneous part of the $D_{\alpha}$ propagator and brings it to the $E_{\alpha}$ propagator, which describes the full physical interaction mediated by the quantized electric field [26].

Our formulation of QED-(TD)DFT combines one of the most popular exact methods for ground (excited) state electronic calculations [27], with the full quantum treatment of the electromagnetic field. In this theory, the wave function of the total system $\Psi\left(\left\{\mathbf{r}_{j}\right\},\left\{q_{\alpha}\right\}, t\right)$ is a unique functional of the electron density $n(\mathbf{r} t)=\langle\Psi|\hat{n}(\mathbf{r})| \Psi\rangle$ and the expectation values of the photon coordinates $Q_{\alpha}(t)=$ $\left\langle\Psi\left|\hat{q}_{\alpha}\right| \Psi\right\rangle[9]$. The former can be calculated for a fictitious Kohn-Sham (KS) system of $N$ noninteracting particles, whose orbitals $\left\{\phi_{j}\right\}$ satisfy the self-consistent equations $\mathrm{i} \partial_{t} \phi_{j}(\mathbf{r} t)=\left[-\nabla^{2} / 2+v_{s}(\mathbf{r} t)\right] \phi_{j}(\mathbf{r} t)$ with the potential $v_{s}=v_{\mathrm{ext}}+v_{\mathrm{Hxc}}^{\mathrm{el}}+v_{\mathrm{eff}}^{\alpha}$. Here, we assume [9] the separate description of the Coulomb interaction $V_{\text {ee }}$ and the photonmediated interaction $W_{\text {ee }}$ by the standard TDDFT Hartreexc term $v_{\mathrm{Hxc}}^{\mathrm{el}}[n]$ and the effective potential $v_{\mathrm{eff}}^{\alpha}\left[n, Q_{\alpha}\right]$. The latter is defined as $v_{\mathrm{eff}}^{\alpha}=v_{\mathrm{MF}}^{\alpha}+v_{\mathrm{xc}}^{\alpha}$, where

$$
v_{\mathrm{MF}}^{\alpha}(\mathbf{r} t)=\int d 1 W_{\mathrm{ee}}^{R}\left(\mathbf{r} t, \mathbf{r}_{1} t_{1}\right) n\left(\mathbf{r}_{1} t_{1}\right)
$$

is the mean-field contribution due to $M$ classical electromagnetic modes, whose expectation values $Q_{\alpha}$ obey the Ampere-Maxwell equation for the displacement field. All the quantum many-body effects are embedded in the unknown xc potential, which must be approximated. Assuming the treatment of the electronic contribution $v_{\mathrm{xc}}^{\mathrm{el}}$ by standard TDDFT functionals (e.g., $x$-only OEP or KLI [13], ALDA, GGA), we generalize the OEP approach to construct approximations to the photonic contribution $v_{\mathrm{xc}}^{\alpha}$.

We derive the TDOEP equation for the electron-photon system starting from the linearized Sham-Schlüter equation on the Keldysh contour [15]

$$
\begin{aligned}
\int & d 2 G_{s}(1,2) v_{\mathrm{xc}}(2) G_{s}(2,1) \\
= & \int d 2 \int d 3 G_{s}(1,2) \Sigma(2,3) G_{s}(3,1),
\end{aligned}
$$

where the electron self-energy $\Sigma$ contains the interaction $W_{\text {ee }}$ of Eq. (3) and $G_{s}$ is the Green's function of the timedependent KS system. Equation (5) allows one to perturbatively construct the local potential $v_{\text {xc }}$, that mimics the effects of the self-energy $\Sigma$, to any desired order in the coupling strength $\lambda_{\alpha}$. Analogously to the $G W$ approximation $[28,29]$ for electronic structure methods, we approximate the electron self-energy by the exchangelike diagram

$$
\Sigma(1,2)=\mathrm{i} G_{s}(1,2) W_{\text {ee }}(2,1),
$$

where we assume the photon propagator in $W_{\text {ee }}$ to be free. Here, the quantum nature of the electromagnetic field is accounted for by the dynamical part of $\Sigma$, related to the first term of Eq. (3). This part describes the processes of emission and absorption of a photon. Neglecting the above dynamical contribution to $v_{\text {eff }}$ corresponds to the classical treatment of the electromagnetic field.

Making use of the Langreth rules [30], we rewrite Eq. (5) more explicitly as follows

$$
\begin{aligned}
& \mathrm{i} \int_{-\infty}^{t} d t_{1} G_{s}^{R}\left(t, t_{1}\right) v_{\mathrm{xc}}\left(t_{1}\right) G_{s}^{<}\left(t_{1}, t\right)+\text { c.c. } \\
& =\mathrm{i} \int_{-\infty}^{t} d t_{1} \int_{-\infty}^{t_{1}} d t_{2} G_{s}^{R}\left(t, t_{1}\right)\left[\Sigma^{>}\left(t_{1}, t_{2}\right) G_{s}^{<}\left(t_{2}, t\right)\right. \\
& \left.\quad-\Sigma^{<}\left(t_{1}, t_{2}\right) G_{s}^{>}\left(t_{2}, t\right)\right]+ \text { c.c. },
\end{aligned}
$$

where the superscripts $R,>$ and $<$ stand for retarded, greater, and lesser Keldysh components, respectively, and the integration over the spatial coordinates is implied. For computational convenience we consider Eq. (7) in the low temperature limit $\mathrm{T} \rightarrow 0$. The electron-photon collision integral on the right-hand side then is responsible for the spontaneous photon emission of the excited electrons and the broadening in the electronic levels. Using Eq. (6) for the self-energy and expressing all $G_{s}$ in terms of KS orbitals [30], Eq. (7) becomes

$$
\begin{aligned}
& \mathrm{i} \sum_{i, j} \int_{-\infty}^{t} d t_{1}\left[\left\langle\phi_{i}\left(t_{1}\right)\left|v_{x}\left(t_{1}\right)\right| \phi_{j}\left(t_{1}\right)\right\rangle f_{i}-S_{i j}\left(t_{1}\right)\right] \phi_{j}^{*}(t) \phi_{i}(t) \\
& \quad+\text { c.c. }=0
\end{aligned}
$$

where we define

$$
\begin{aligned}
S_{i j}\left(t_{1}\right)= & \sum_{k, \alpha} \int_{-\infty}^{t_{1}} d t_{2} d_{i k}^{\alpha}\left(t_{2}\right) d_{k j}^{\alpha}\left(t_{1}\right)\left[\left(1-f_{i}\right) f_{k} \mathcal{W}^{>}\left(t_{1}, t_{2}\right)\right. \\
& \left.-f_{i}\left(1-f_{k}\right) \mathcal{W}^{<}\left(t_{1}, t_{2}\right)\right]
\end{aligned}
$$

with $\quad \mathcal{W}^{\gtrless}\left(t_{1}, t_{2}\right)=\omega_{\alpha}^{2}\left(-\mathrm{i} / 2 \omega_{\alpha}\right) e^{ \pm \mathrm{i} \omega_{\alpha}\left(t_{2}-t_{1}\right)} \pm \delta\left(t_{1}-t_{2}\right)$. Here, $f_{i}$ is the fermion occupation number and $d_{i k}^{\alpha}(t)=$ $\lambda_{\alpha}\left\langle\phi_{i}(t)|\mathbf{r}| \phi_{k}(t)\right\rangle$ is the dipole matrix element projected on the coupling constant of the $\alpha$ mode. For definiteness we assume that the external potential $v_{\text {ext }}$ does not depend on time for all $t<0$. Hence the orbitals $\left\{\phi_{j}\right\}$ are solutions of the KS equations with the initial condition $\phi_{j}(\mathbf{r} t)=$ $\phi_{j}(\mathbf{r}) e^{-\mathrm{i} \varepsilon_{j} t}$ for $-\infty<t \leq 0$. Equation (8) relates the matrix 
elements of $v_{x}$ to the matrix elements $S_{i j}$ of the self-energy $\Sigma$, and thus to the physical processes of excitation (annihilation) of electron-hole pairs by photon absorption (emission). Alternatively, Eq. (8) can be derived via variational principle from the Keldysh action functional, with the exchange part given by

$$
\begin{aligned}
A_{x}= & \sum_{i, k, \alpha} \int d z_{1} \int d z_{2} d_{i k}^{\alpha}\left(z_{2}\right) d_{k i}^{\alpha}\left(z_{1}\right)\left(1-f_{i}\right) f_{k} \\
& \times \theta\left(z_{1}-z_{2}\right)\left[\omega_{\alpha}^{2}\left(\frac{-\mathrm{i}}{2 \omega_{\alpha}}\right) e^{\mathrm{i} \omega_{\alpha}\left(z_{2}-z_{1}\right)}+\delta\left(z_{1}-z_{2}\right)\right],
\end{aligned}
$$

where $z$ denotes the contour variable. Furthermore, the time-dependent mean-field potential is evaluated from Eq. (4) as

$$
\begin{aligned}
v_{\mathrm{MF}}(\mathbf{r} t)= & -\sum_{\alpha} \omega_{\alpha}\left(\boldsymbol{\lambda}_{\alpha} \mathbf{r}\right) \int_{0}^{t} d t_{1} \sin \left[\omega_{\alpha}\left(t-t_{1}\right)\right]\left[\boldsymbol{\lambda}_{\alpha} \mathbf{R}\left(t_{1}\right)\right] \\
& -\sum_{\alpha}\left(\boldsymbol{\lambda}_{\alpha} \mathbf{r}\right)\left\{\left[\boldsymbol{\lambda}_{\alpha} \mathbf{R}(0)\right] \cos \left(\omega_{\alpha} t\right)-\left[\boldsymbol{\lambda}_{\alpha} \mathbf{R}(t)\right]\right\},
\end{aligned}
$$

where $\mathbf{R}(t)=\int d^{3} \mathbf{r r} n(\mathbf{r} t)$ is the expectation value of the dipole moment operator of the electronic system.

In the case of time independent external potential, Eq. (8) reduces to the stationary OEP equation for the equilibrium electron-photon system

$$
\sum_{i, j}\left[\frac{\left\langle\phi_{i}\left|v_{x}\right| \phi_{j}\right\rangle}{\varepsilon_{i}-\varepsilon_{j}-\mathrm{i} \eta} f_{i}-S_{i j}\right] \phi_{j}^{*}(\mathbf{r}) \phi_{i}(\mathbf{r})+\text { c.c. }=0,
$$

where

$$
\begin{aligned}
S_{i j}= & \sum_{k, \alpha} \frac{d_{i k}^{\alpha} d_{k j}^{\alpha}\left(\varepsilon_{i}-\varepsilon_{k}-\mathrm{i} \eta\right)}{2\left(\varepsilon_{i}-\varepsilon_{j}-\mathrm{i} \eta\right)}\left[\frac{f_{i}\left(1-f_{k}\right)}{\varepsilon_{i}-\varepsilon_{k}-\omega_{\alpha}-\mathrm{i} \eta}\right. \\
& \left.+\frac{\left(1-f_{i}\right) f_{k}}{\varepsilon_{i}-\varepsilon_{k}+\omega_{\alpha}-\mathrm{i} \eta}\right] .
\end{aligned}
$$

Here, we assume the limit $\eta \rightarrow 0$. Apparently, Eq. (11) describes virtual processes of excitation of electron-hole pairs, supplemented by the emission of photons. Variationally Eq. (10) can be derived employing the second-order correction to the ground-state energy

$$
E_{x}=-\frac{1}{2} \sum_{i, k, \alpha}\left|d_{i k}^{\alpha}\right|^{2}\left\{\omega_{\alpha} \frac{\left(1-f_{i}\right) f_{k}}{\varepsilon_{i}-\varepsilon_{k}+\omega_{\alpha}}-\left(1-f_{i}\right) f_{k}\right\},
$$

which is the Lamb shift due to the virtual emission of photons [31]. The second term in Eq. (12) comes from the term $\sum_{\alpha}\left(\boldsymbol{\lambda}_{\alpha} \mathbf{R}\right)^{2} / 2$ in the Hamiltonian, and accounts for the free electron behavior in the high photon energy limit $\omega_{\alpha} \rightarrow \infty$.
As a proof of principles, we apply these results to a simple, yet nontrivial quantum optical model that can be solved exactly, i.e., the tight-binding model for the $\mathrm{H}_{2}^{+}$ molecule coupled to one photon mode. The one electron choice here prevents from introducing the extra error in approximating the standard TDDFT potential $v_{\mathrm{xc}}^{\mathrm{el}}$, thus allowing us to assess the accuracy of our approximation to the electron-photon potential $v_{\mathrm{xc}}$. The electron coordinate can only take two values, given by the atomic site indexes $(1,2)$. The electron density reduces to the on-site occupation number $n_{1,2}$. By projecting the Hamiltonian in Eq. (1) onto the two-site space, the electronic kinetic energy gives the tunneling amplitude between the sites. As the total occupation is fixed, the external and photon fields couple to the on-site occupation difference $\Delta n=n_{1}-n_{2}$. This plays the role of the TDDFT density variable for the model. In terms of Pauli matrices $\sigma_{x, z}\left[\hat{T} \rightarrow-T \hat{\sigma}_{x}\right.$ and $\left.\Delta \hat{n} \rightarrow \hat{\sigma}_{z}\right]$, the projected Hamiltonian

$$
\begin{aligned}
\hat{H}= & -T \hat{\sigma}_{x}+\left[\sqrt{\frac{\omega}{2}} \lambda\left(\hat{a}+\hat{a}^{\dagger}\right)+v_{\mathrm{ext}}(t)\right] \hat{\sigma}_{z} \\
& +\omega\left(\hat{a}^{\dagger} \hat{a}+\frac{1}{2}\right)+\frac{\lambda^{2}}{2}
\end{aligned}
$$

reads isomorphic to the Rabi Hamiltonian with external potential $v_{\text {ext }}(t)$ and coupling strength $\sqrt{\omega / 2} \lambda$.

We consider first the system in equilibrium. The summation in Eq. (10) runs over the ground $(g)$ and excited $(e)$ KS states with the corresponding orbitals, $\phi_{g}^{\dagger}=(\bar{v}, \bar{u})$ and $\phi_{e}^{\dagger}=(\bar{u},-\bar{v})$, and eigenvalues, $\varepsilon_{g}=-W$ and $\varepsilon_{e}=W$. Here, $\quad \bar{u}, \bar{v}=\sqrt{\left(1 \pm v_{s} / W\right) / 2}$ and $W=\sqrt{v_{s}^{2}+T^{2}}$. Explicitly, Eq. (10) gives

$$
v_{x}=-\lambda^{2} \frac{v_{s}}{W}\left[\frac{\omega(\omega+3 W)}{(\omega+2 W)^{2}}-1\right],
$$

where the second term corresponds to the classical contribution associated with the first interaction term in Eq. (3). The total energy functional takes the form

$$
E\left[v_{s}\right]=-T\left\langle\sigma_{x}\right\rangle+v_{\mathrm{ext}} \Delta n+E_{x}\left[v_{s}\right]+\frac{1}{2} \omega,
$$

where $\Delta n=-v_{s} / W$ and Eq. (12) reduces to

$$
E_{x}=\frac{\lambda^{2} T^{2}}{W(\omega+2 W)} .
$$

The Lamb shift of Eq. (16) vanishes in the classical limit of coupling $\lambda \rightarrow \infty$, as expected.

In Fig. 1 we show the calculated OEP density difference $\Delta n$ and total energy $E$ as functions of the coupling strength $\lambda$, compared to the results from the exact and classical treatment of the electromagnetic field. It is worth noting that $0.1 \lesssim \lambda \lesssim 1.4$ and $\lambda \gtrsim 1.4$ corresponds, respectively, to 


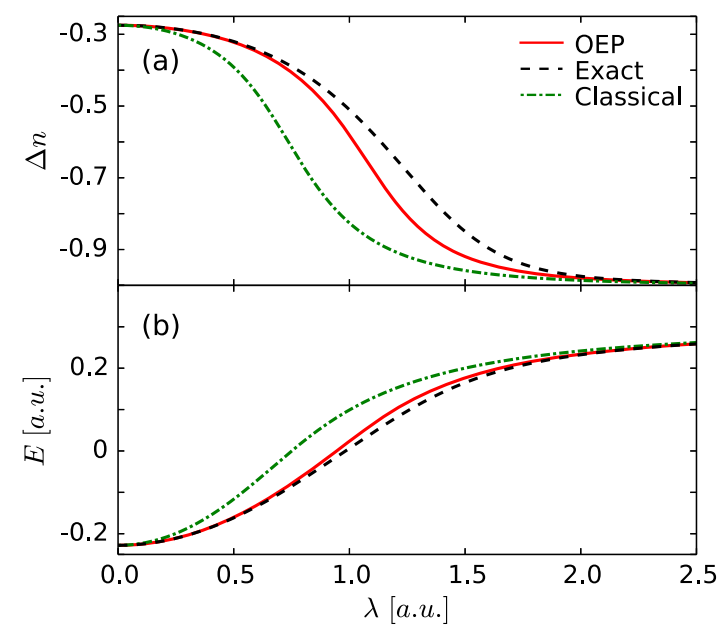

FIG. 1 (color online). Comparison of the OEP (red), exact (black), and classical (green) (a) density difference $\Delta n$ and (b) energy $E$ versus the coupling parameter $\lambda$ in a.u. Other parameters: $\omega=1, v_{\text {ext }}=0.2, T=0.7$.

ultrastrong [32] and deep strong coupling [33] values. The eigenvalue problem for the static Rabi Hamiltonian in Eq. (13) is solved by employing the exact diagonalization technique [34,35], after proper truncation of the Fock space. We observe that both the OEP and classical approximations reproduce qualitatively the electron's confinement on the excited level, as the shift in the energy levels increases with the coupling strength, and recover the exact result in the limit $\lambda \rightarrow \infty$. In addition, our OEP scheme is by construction exact in the weak coupling regime. For the density difference $\Delta n$ shown in (a), we see excellent agreement between the OEP and the exact results up to $\lambda=0.7$ and above $\lambda=2$. On the contrary, the classical result is only asymptotically accurate and largely underestimating in between. Regarding the energies $E$ shown in (b), the OEP energy is close to the exact values in the whole coupling range, with only small deviations around $\lambda=1.3$. In contrast, the classical approximation performs reliably only in the limits of very small or very high interaction strength.

The TDOEP Eq. (8) for the Rabi model simplifies to

$$
\begin{aligned}
& \mathrm{i} \int_{-\infty}^{t} d t_{1} \tilde{v}_{x}\left(t_{1}\right) d_{g e}\left(t_{1}\right) d_{e g}(t)+\text { c.c. } \\
& \quad=\lambda^{2} \omega \int_{-\infty}^{t} d t_{1} \int_{-\infty}^{t_{1}} d t_{2} c\left(t, t_{1}\right) d_{e g}\left(t_{2}\right) e^{\mathrm{i} \omega\left(t_{2}-t_{1}\right)}+\text { c.c. },
\end{aligned}
$$

where $\tilde{v}_{x}=v_{x}(t)+\lambda^{2} \Delta n(t), \quad d_{g e}(t)=\left\langle\phi_{g}(t)\left|\hat{\sigma}_{z}\right| \phi_{e}(t)\right\rangle$, and $c\left(t, t_{1}\right)=d_{g e}(t) \Delta n\left(t_{1}\right)-d_{g e}\left(t_{1}\right) \Delta n(t)$. Moreover, the mean-field potential of Eq. (9) explicitly reads as

$$
\begin{aligned}
v_{\mathrm{MF}}(t)= & -\lambda^{2} \omega \int_{0}^{t} d t_{1} \sin \left[\omega\left(t-t_{1}\right)\right] \Delta n\left(t_{1}\right) \\
& -\lambda^{2} \Delta n \cos (\omega t)+\lambda^{2} \Delta n(t) .
\end{aligned}
$$

Employing the numerical algorithm presented in Ref. [36], we solve Eq. (17) self-consistently for $t>0$, together with the time-dependent KS equation. The former, which is a Volterra integral equation of the first kind, is evaluated using a midpoint integration scheme combined with the trapezoidal rule [37]. The latter is propagated with a predictor-corrector scheme using an exponential midpoint propagator [38]. In Fig. 2 we compare the time evolution of the calculated OEP density difference $\Delta n$ and effective potential $v_{\text {eff }}$ with the exact and classical results, approaching the ultrastrong coupling regime in two different setups. In the first setting, we assume that the electron-photon system, interacting with coupling constant $\lambda=0.1$, is driven out of equilibrium at $t=0$ by a sudden switch in the external perturbation $v_{\text {ext }}(t)=-0.2 \operatorname{sgn}(t)$. In the second configuration, we choose a noninteracting initial state with $v_{\text {ext }}(t)=0$, while switching on at later times the electron-photon coupling $\lambda(t)=0.1 \theta(t)$. Here, we use as the initial state for the propagation $|\Psi\rangle=$ $(1 / 2|1\rangle+\sqrt{3} / 2|2\rangle) \otimes|0\rangle$, where $|1\rangle$ and $|2\rangle$ are the basis vectors of the electron system, and $|0\rangle$ is the photon vacuum field. For the chosen parameters, the various density differences in the two setups undergo off-resonant Rabi oscillations with nearly identical relative behavior. The errors $\delta \Delta n$ in the OEP and classical density difference are shown in (a) for the sudden-switch example. The first is remarkably low in the entire coupling range. The second is about $10 \%$ at $t=20$ a.u. and increases up to $20 \%$ at $t=40$ a.u. The quantum contribution to the OEP is given by the right-hand side of Eq. (17) and its role in the Rabi

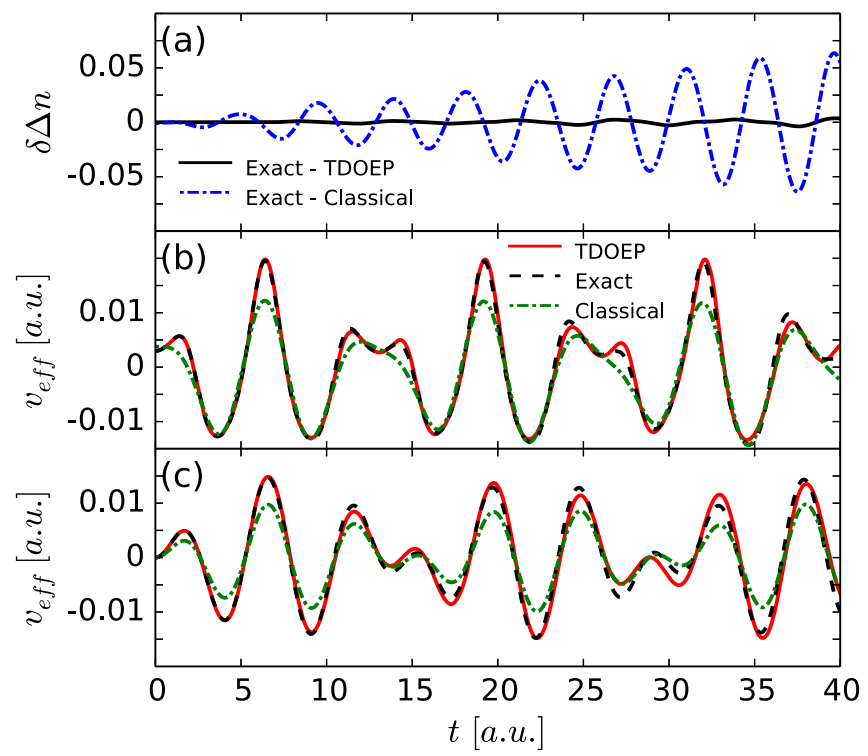

FIG. 2 (color online). Comparison of the (a) errors $\delta \Delta n$ in the TDOEP (black) and classical (blue) density difference $\Delta n$ and (b),(c) TDOEP (red), exact (black), and classical (green) effective potential $v_{\text {eff }}$ versus time $t$ in a.u. for the configurations: (a),(b) $v_{\text {ext }}=-0.2 \operatorname{sgn}(t), \lambda=0.1$ and (c) $v_{\text {ext }}=0, \lambda=0.1 \theta(t)$. Other parameters: $\omega=1, T=0.7$. 
oscillations is essentially quantified by the error in the classical density difference. Significant is also the improvement of the OEP approach against the classical approximation in the effective potential. It should be noted that, unlike the density difference, this doesn't correspond to a physical observable. As we can see in (b) for the suddenswitch case, and in (c) for the noninteracting initial configuration, the TDOEP result is very accurate up to $t=20$ a.u. At later times, small deviations appear, especially in (c), where the potential shows a more complex dynamics. Nevertheless, the improvement with respect to the classical result is still evident.

In conclusion, a first-principles approach for describing the quantum dynamics of realistic many-electron systems interacting with photons is proposed. For methodological purposes we have considered here its application to the off-resonant Rabi model. It has been shown that already the lowest order (TD)OEP for the model gives accurate stationary and dynamical properties far beyond the weakcoupling regime, clearly improving over the classical treatment of the electromagnetic field. Future developments include simplifying the QED-TDOEP scheme along the lines of the TDKLI approximation [13,39]. This work opens the path to a simplified numerical description of novel phenomena at the interface between condensed matter physics and quantum optics.

We acknowledge financial support by the European Research Council Advanced Grant DYNamo (ERC2010-AdG-267374), European Commission project CRONOS (Grant No. 280879-2 CRONOS CP-FP7), Spanish Grant (FIS2013-46159-C3-1-P), Ikerbasque and Grupo Consolidado UPV/EHU del Gobierno Vasco (IT578-13).

*camilla.pellegrini@ehu.es

†flick@fhi-berlin.mpg.de

*ilya.tokatly@ehu.es

§appel@fhi-berlin.mpg.de

angel.rubio@ehu.es

[1] J. P. Reithmaier, G. Sęk, A. Löffler, C. Hofmann, S. Kuhn, S. Reitzenstein, L. V. Keldysh, V. D. Kulakovskii, T. L. Reinecke, and A. Forchel, Nature (London) 432, 197 (2004).

[2] A. A. Houck, H. E. Türeci, and J. Koch, Nat. Phys. 8, 292 (2012).

[3] A. F. van Loo, A. Fedorov, K. Lalumière, B. C. Sanders, A. Blais, and A. Wallraff, Science 342, 1494 (2013).

[4] K.-J. Boller, A. Imamoglu, and S. E. Harris, Phys. Rev. Lett. 66, 2593 (1991).

[5] M. S. Tame, K. R. McEnery, Ş. K. Özdemir, J. Lee, S. A. Maier, and M. S. Kim, Nat. Phys. 9, 329 (2013).

[6] J. T. Barreiro, M. Müller, P. Schindler, D. Nigg, T. Monz, M. Chwalla, M. Hennrich, C. F. Roos, P. Zoller, and R. Blatt, Nature (London) 470, 486 (2011).

[7] A. Fontcuberta i Morral and F. Stellacci, Nat. Mater. 11, 272 (2012).
[8] J. A. Hutchison, T. Schwartz, C. Genet, E. Devaux, and T. W. Ebbesen, Angew. Chem., Int. Ed. 51, 1592 (2012).

[9] I. V. Tokatly, Phys. Rev. Lett. 110, 233001 (2013).

[10] M. Ruggenthaler, J. Flick, C. Pellegrini, H. Appel, I. V. Tokatly, and A. Rubio, Phys. Rev. A 90, 012508 (2014).

[11] M. Farzanehpour and I. V. Tokatly, Phys. Rev. B 90, 195149 (2014).

[12] M. Ruggenthaler, F. Mackenroth, and D. Bauer, Phys. Rev. A 84, 042107 (2011).

[13] C. A. Ullrich, U. J. Gossmann, and E. K. U. Gross, Phys. Rev. Lett. 74, 872 (1995).

[14] C. A. Ullrich, U. J. Gossmann, and E. K. U. Gross, Ber. Bunsenges. Phys. Chem. 99, 488 (1995).

[15] R. van Leeuwen, Phys. Rev. Lett. 76, 3610 (1996).

[16] A. Görling, Phys. Rev. A 55, 2630 (1997).

[17] S. Kümmel and J. P. Perdew, Phys. Rev. Lett. 90, 043004 (2003).

[18] S. Kümmel and L. Kronik, Rev. Mod. Phys. 80, 3 (2008).

[19] B. W. Shore and P. L. Knight, J. Mod. Opt. 40, 1195 (1993).

[20] D. Braak, Phys. Rev. Lett. 107, 100401 (2011).

[21] F. A. Wolf, F. Vallone, G. Romero, M. Kollar, E. Solano, and D. Braak, Phys. Rev. A 87, 023835 (2013).

[22] The derivation can be generalized to the case of atom-field coupling beyond the dipole approximation in a straightforward manner.

[23] C. Cohen-Tannoudji, J. Dupont-Roc, and G. Grynberg, Atom-Photon Interactions (Wiley-VCH, Weinheim, 2004).

[24] D. P. Craig and T. Thirunamachandran, Molecular Quantum Electrodynamics (Academic Press, London, 1984).

[25] We use atomic units (a.u.) throughout the Letter.

[26] In the frequency domain the effective interaction of Eq. (3) reads $\mathcal{W}_{\alpha}(\omega)=\omega_{\alpha}^{2} /\left(\omega^{2}-\omega_{\alpha}^{2}\right)+1=\omega^{2} /\left(\omega^{2}-\omega_{\alpha}^{2}\right) \sim\left\langle E_{\alpha} ; E_{\alpha}\right\rangle_{\omega}$. As it should be, the total physical interaction is proportional to $\omega^{2}$ because only accelerated electrons can emit electromagnetic radiation.

[27] M. A. L. Marques, C. A. Ullrich, F. Nogueira, A. Rubio, K. Burke, and E. K. U. Gross, Time-Dependent DensityFunctional Theory, 706 (Springer, Berlin, 2010).

[28] L. Hedin, Phys. Rev. 139, A796 (1965).

[29] L. Onida, L. Reining, and A. Rubio, Rev. Mod. Phys. 74, 601 (2002).

[30] G. Stefanucci and R. van Leeuwen, Nonequilibrium ManyBody Theory of Quantum Systems (Cambridge University Press, Cambridge, England, 2013).

[31] A. N. Mitra, Resonance 10, 33 (2005).

[32] T. Niemczyk, F. Deppe, H. Huebl, E. P. Menzel, F. Hocke, M. J. Schwarz, J. J. Garcia-Ripoli, D. Zueco, T. Hümmer, E. Solano et al., Nat. Phys. 6, 772 (2010).

[33] A. Crespi, S. Longhi, and R. Osellame, Phys. Rev. Lett. 108, 163601 (2012).

[34] A. I. Streltsov, O. E. Alon, and L. S. Cederbaum, Phys. Rev. A 81, 022124 (2010).

[35] J. Flick, H. Appel, and A. Rubio, J. Chem. Theory Comput. 10, 1665 (2014).

[36] H. O. Wijewardane and C. A. Ullrich, Phys. Rev. Lett. 100, 056404 (2008).

[37] P. Linz, Computer Journal 12, 393 (1969).

[38] A. Castro, M. A. L. Marques, and A. Rubio, J. Chem. Phys. 121, 3425 (2004).

[39] C. Schäfer, H. Appel, J. Flick, C. Pellegrini, I. V. Tokatly, and A. Rubio (unpublished). 